\title{
Analysing spatial interdependence among the 2011 Thailand flood-affected small and medium enterprises for reduction of disaster recovery time period
}

\author{
Shubham Pathak ${ }^{1 *}$ and Jorge Chica Olmo²
}

\begin{abstract}
Natural disasters have been a significant hurdle in the economic growth of middle-income developing countries. Thailand has also been suffering from recurring flood disasters and was most which are severely affected during the 2011 floods. This paper aims to identify the various factors that impact the speed of disaster recovery among the Small and Medium Enterprises (SMEs) severely affected by the floods in Pathumthani province in central Thailand, and how it is related to its speed decision of neighbours SMEs. The methodology adopts a spatial econometric model, to analysis and understand each of the chosen factors' impact. The findings include the impact of disaster resilience, mitigation and planning at the SME level as well as the government level. The absence of accurate perception of actual risk, flood insurance and disaster management planning before the 2011 floods had contributed to the severity of the impacts during the 2011 floods.
\end{abstract}

Keywords: Floods, Small and medium enterprises (SMEs), Spatial econometrics and disaster recovery

\section{Introduction and literature review}

Natural disasters are inevitable and adversely impact the social, environmental and economic setup wherever they strike. These adverse impacts are dependent upon several factors which contribute towards the severity of a disaster event. Floods in Thailand has been a major threat towards the Small and Medium Enterprises (SMEs) and communities residing in flood plains (Pathak and Ahmad 2016). The severe impacts of the 2011 floods not only halted the Thai economy, still, they had effects on global economy due to lack of supply of manufacturing parts such as hard disk drives and accessories for motorised vehicles (Haraguchi and Lall 2015). This paper aims at analysing the factors affecting the speed of disaster recovery among these flood affected

\footnotetext{
* Correspondence: shubhampathak@gmail.com; shubham.pa@mail.wu.ac.th 'Logistics Analytics and Supply Chain Management, Walailak University International College, Walailak University, Tha Sala, Nakhon Si Thammarat, Thailand

Full list of author information is available at the end of the article
}

SMEs in Thailand. The spatial econometrics approach has been undertaken to utilize the scarce resources available with these SMEs in order to improved manage the future flood events (Zhang 2016).

Previous literature regarding floods and SMEs in Thailand pertaining to how they deal with the disaster management and recovery process in a disaster scenario. Previous literature focuses on the disaster recovery and rehabilitation of the SMEs. However, there is lack of research pertaining to the neighbouring SMEs (Cravo et al. 2015).

Spatial econometrics deals with the combination of spatial analysis along with econometrics and provide for the estimated and analysed parameters using regression analysis. Previous literature focused on the cross sectional dependence at local as well as global level (Elhorst et al. 2018), however, less has been researched in terms of SMEs vulnerable from natural disasters. Spatial econometrics is important when the collected data deals 
with the location component and spatial dependence between the data collected (Lesage 1999). The economics of disasters have been gaining interest in the literature in the recent decades (Yu and Tang 2017; Rikimaru and Shibata 2017).

Spatial econometrics have been researched in accordance with the neighbouring agents and their behaviours in terms of economic and managerial decision making (Lesage 1999). The models have been dealing with the variables show the spatial dependence among them and in relation to the independent variable. However, there is a lack of data among the SMEs on the repercussion of natural disaster. This paper emphasis to explore the spatial dependence among variables of the flood vulnerable SMEs in Thailand. In terms of spatial location, SMEs in Thailand has been scattered in the central provinces. One of the major reasons of larger impacts upon SMEs from floods is the lack of spatial analysis which deprives them of crucial information such as the vulnerability of their physical location and exposure towards floods (Shafi et al. 2020). Therefore, this paper aims at combining economic and spatial aspects of 2011 floods among the affected neighbouring SMEs in Thailand.

Previous literature provides for several studies to post disaster recovery period being affected by endogenous and exogenous factors. However, in econometrics the selected factors are exogenous. The factors selected for this study are divided into primary and secondary factors. The primary factors include the characteristics of business owners/managers and business enterprises (Marshall et al. 2015; Ezgi 2016). The secondary factors involve the impact of disasters, economic turmoil and the measures and aid provided after a disaster event (Sydnor et al. 2017; Khan and Sayem 2013).

However, emphasis upon neighboring SMEs and their disaster recovery time period has not been studies in a quantified research. The absence of spatial dependence or interdependence among the SMEs would lead to inadequate and ineffective disaster recovery planning at various stages of disaster governance (Li and Hong 2019).

\section{Floods in Thailand}

Floods are a common and recurring disaster in Thailand. Major floods were recorded throughout the decades of 90's and 2000's. However, 2011 floods during the months of October to December 2011, was one of the most destructive floods in terms of economic and social losses and damages in recent decades. With the increase of population and growth of the Thai economy the impacts of floods in terms of social and economic terms have been increasing tremendously in the recent years.

The 2011 floods were among the costliest disasters in Thailand (Government and Bank 2012). The economic losses were escalated to over 1.4 Trillion Thai Baht
(42 Billion USD) and an additional recovery expenses of over 1.5 Trillion Thai Baht (45 Billion USD) since the 2011 floods (Khaikhan and James 2019). It submerged almost entire central region of Thailand bringing all the activities at a standstill for over a period of one to three months. Some provinces including Ayutthaya and Pathumthani were severely affected due to presence of large number of business enterprises which were inundated in over $2.5 \mathrm{~m}$ of flood waters.

. The Thai SMEs provide for $70 \%$ of the employment and contributes $45 \%$ (215 billion US\$) towards the total of Thai National Gross Domestic Product (GDP) and International Trade (OSMEP 2018). The Office of Small and Medium Enterprises Promotion (OSMEP) has been striving hard in maintaining SME's growth and development in Thailand (OSMEP, 2011). The office analyses the constant trends of SME's growth and contribution towards the GDP and achieving sustainable SMEs. However, lack of cooperation between other departments including OSMEP and disaster management departments hampers the efforts to support the SMEs throughout and afterward floods or disaster scenarios (Suvittawat 2019).

Defining SMEs is difficult as the definitions varies in different countries and societal setups. The previous literature attempts to explore the definitions and extent of SMEs in Thailand. Therefore, the OSMEP defines the SMEs in Thailand; as all enterprises which have a fixed capital of not more than 100 million Thai baht and employ under 200 hundred employees (OSMEP 2018). Mainly these SMEs deals in manufacturing and service industries (Refer Fig. 1).

The other sectors include retailing and wholesale. However, the manufacturing industries were severely affected with longer recovery time with production halted for over two months and reduced sales in the market in the first quarter of 2012. Several previous researches sheds light on the absence of preparedness and mitigation measures among the vulnerable SMEs in Thailand (Khaikhan and James 2019). One of the major factor was the absence of Insurance amongst the flood vulnerable SMEs, especially in low lying areas of Pathumthani and Ayutthaya provinces in the central Thailand.

\section{Disaster recovery time period}

Accurate and successful disaster management paves way for the faster recovery from an uncertain disaster event (Horney et al. 2016; Coquet et al. 2018). Faster disaster recovery time period depends upon several factors including the management at SME level as well as the government level.

Post disaster recovery period for the business enterprises is essential to ensure sustainable growth and development of any economy (Xiao and Peacock 2014). 


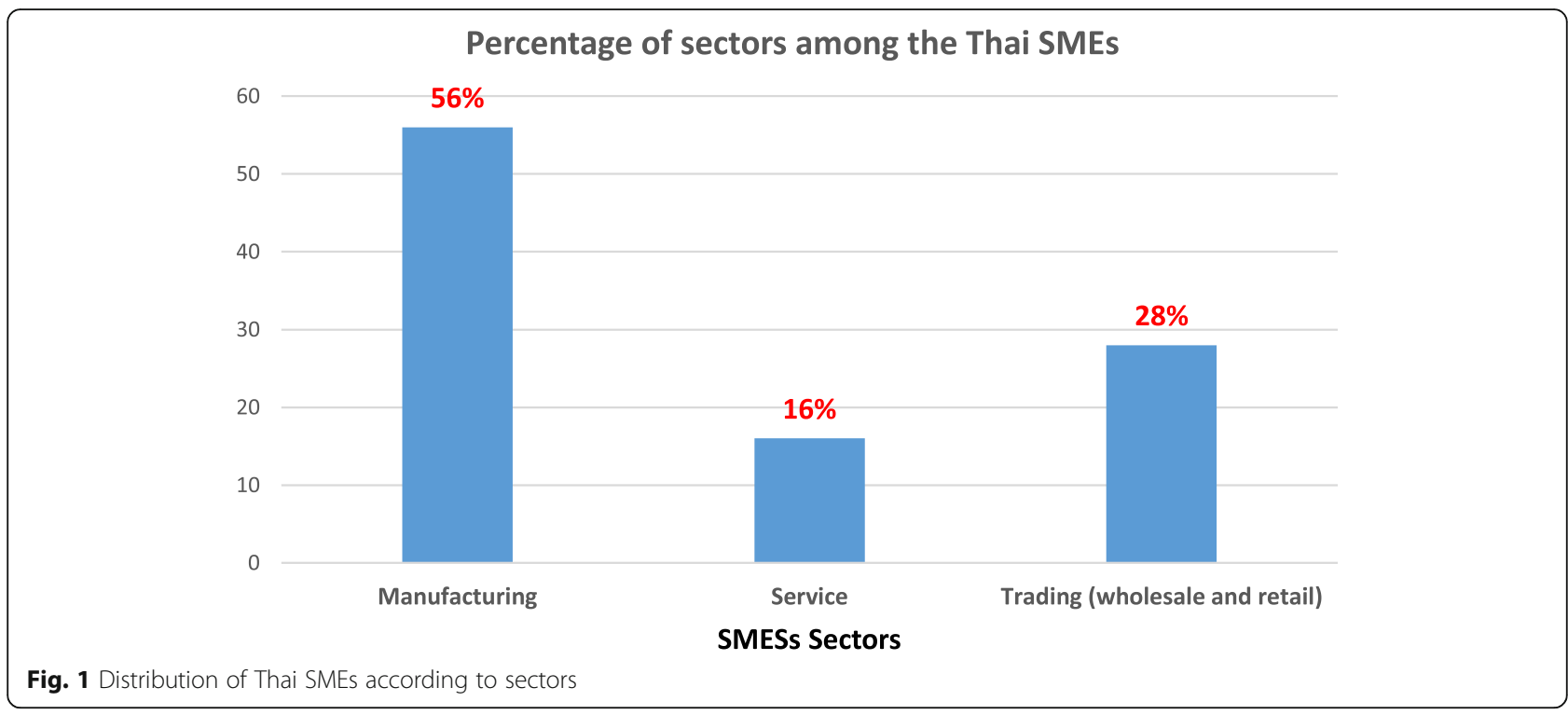

Thus, the recognition towards the Post disaster recovery period has been increasing among the community resilience and sustainability studies (Zhang et al. 2009). The spatial econometrics among neighboring SMEs could be utilized to shorten and reduce the impacts of longer disaster recovery time periods. However, spatial correlations and decision making among the neighboring SMEs have been overlooked in the disaster recovery studies ( $\mathrm{Li}$ and Hong 2019). Therefore, this study aims to understand and analyze the interlinkage amongst the decision making of neighboring business enterprises during and after disasters. The 2011 Thailand floods has been undertaken as the case of disaster event which resulted in huge social-economic damages and losses among SMEs in Thailand.

\section{Methodology}

This work involved a spatial econometric model which was utilized to explain Disaster Recovery Time Period (DRTP), which was dependent upon the data collected among the respondents. The conventional estimation approaches, for example ordinary least squares (OLS), which assumes interdependence among several observations gathered during the data collection process (Pace and LeSage 2009). Nevertheless, spatial data is characterized because there is spatial dependence or interdependence among observations. The spatial econometric models contemplates and analyses the observed spatial interdependence, resulting with the existence of spatial interdependence and autocorrelation (Anselin 1988).

To determine the spatial autocorrelation, Moran's test was used (Moran 1950). The test calculates the ratio amongst cross-product of deviations from mean of variable of interest and their corresponding spatial intervals, along with square of assessed deviations:

$$
I=\frac{n}{\sum_{i=1}^{n} \sum_{j=1}^{n} w_{i j}} \frac{\sum_{i=1}^{n} \sum_{j=1}^{n} w_{i j}\left(y_{i}-\bar{y}\right)\left(y_{j}-\bar{y}\right)}{\sum_{i=1}^{n}\left(y_{i}-\bar{y}\right)^{2}}
$$

whereas yi depicts $\mathrm{i}$-th observation for the variable of DRTP, $\bar{y}$ depicts the mean, $w_{i j}$ depicts spatial weights.

Several methods existing towards modelling the consequences of geographic setting in a spatial econometric model (Elhorst 2014) but traditionally there are two approaches:

\section{The first approach involves the spatial auto regressive (SAR) also known as spatial lag model}

$$
y=\rho W y+\alpha+X \beta+\varepsilon
$$

\section{The second approach includes spatial error model (SEM)}

$$
\begin{aligned}
& y=\alpha+X \beta+u \\
& u=\lambda W u+\varepsilon
\end{aligned}
$$

where $y$ is the values of DRTP; $\alpha$ is the intercept; $\mathrm{X}$ is the explanatory variables $\left(x_{k}\right) \beta$ is the coefficient of explanatory variables; $W$ is the spatial weights matrix, which represents the spatial relationships between neighbors; $W y$ is $W$ multiplied by $y$; $\rho$, shows spatial dependence (Wall 2004); $W u$ is $W$ multiplied by $u ; \lambda$ is the coefficient of $W u$, and $\varepsilon$ is a normal vector perturbations i.i.d.

To determine which of these two models (SAR or SEM) would be more appropriate, the Lagrange Multiplier tests (LM) were analyzed in terms of ascertaining 
Error Dependence (LM-err) along with endogenous spatially lagging (LM-lag) dependent variables. However, both statistics were found to be significant (Anselin et al. 1996), hence LM-err along with the LM-lag are utilized. Once again, they were found to be significant, therefore the model matching with the statistic with the lesser $p$ value were carefully chosen.

A matter of specific concern in a spatial econometric model; is calculation of the interdependence and relationship between the explanatory variable $x_{k}$ and dependent variable. This relationship is directly affected by the geographical location $j$ and it is depicted by the coefficient $\beta_{k}$ in an OLS model. However, the direct effect is equal to $(I-\rho W)^{-1} \beta_{k}$ in a SAR model (LeSage 2008). Similarly, the indirect effect is also analysed among the dependent variable and the neighbouring locations with variations in, $x_{k}$, of location $i$. The total impact is determined by summing the direct and indirect impacts.

\section{Study area and data}

\section{Study area}

The study area was intentionally selected in the Pathumthani province in central Thailand, as it was among the top provinces which severely affected by 2011 floods. The primary data collection was among the 177 SMEs selected randomly on based on the provincial data. These 177 SMEs were geotagged into ArcGIS software and a unique id has been assigned to each of the SMEs (Refer Figs. 2 and 3).

\section{Variables and data}

Several variables were shortlisted after analysis of the previous literature and amongst SMEs located in Thailand. The variables (Refer Table 1) includes the selected variables for this study to analyse each of their association with disaster recovery. Table 1 shows the variables selected.

These factors have been comprehensively considered by several previous researchers (Marshall et al. 2015), however these factors were carefully chosen and segregated according to the socioeconomic and political set up. The nature of governance in Thailand is quite different from several middle income countries around the globe. The 2011 floods in Thailand was also considered to be one of the most severe and disastrous event in recent history of Thailand. The government and SMEs need to understand such scenarios and reach towards

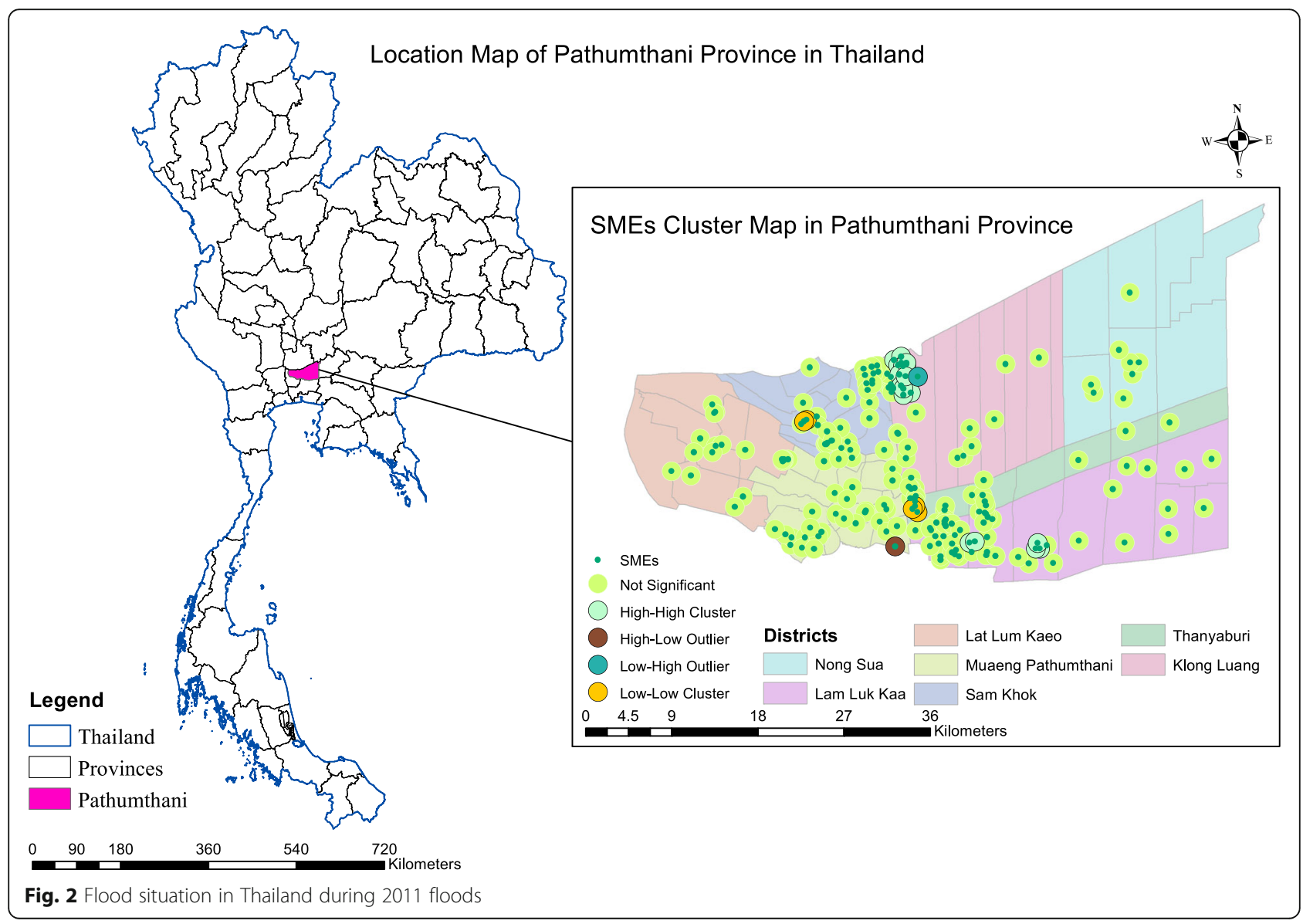




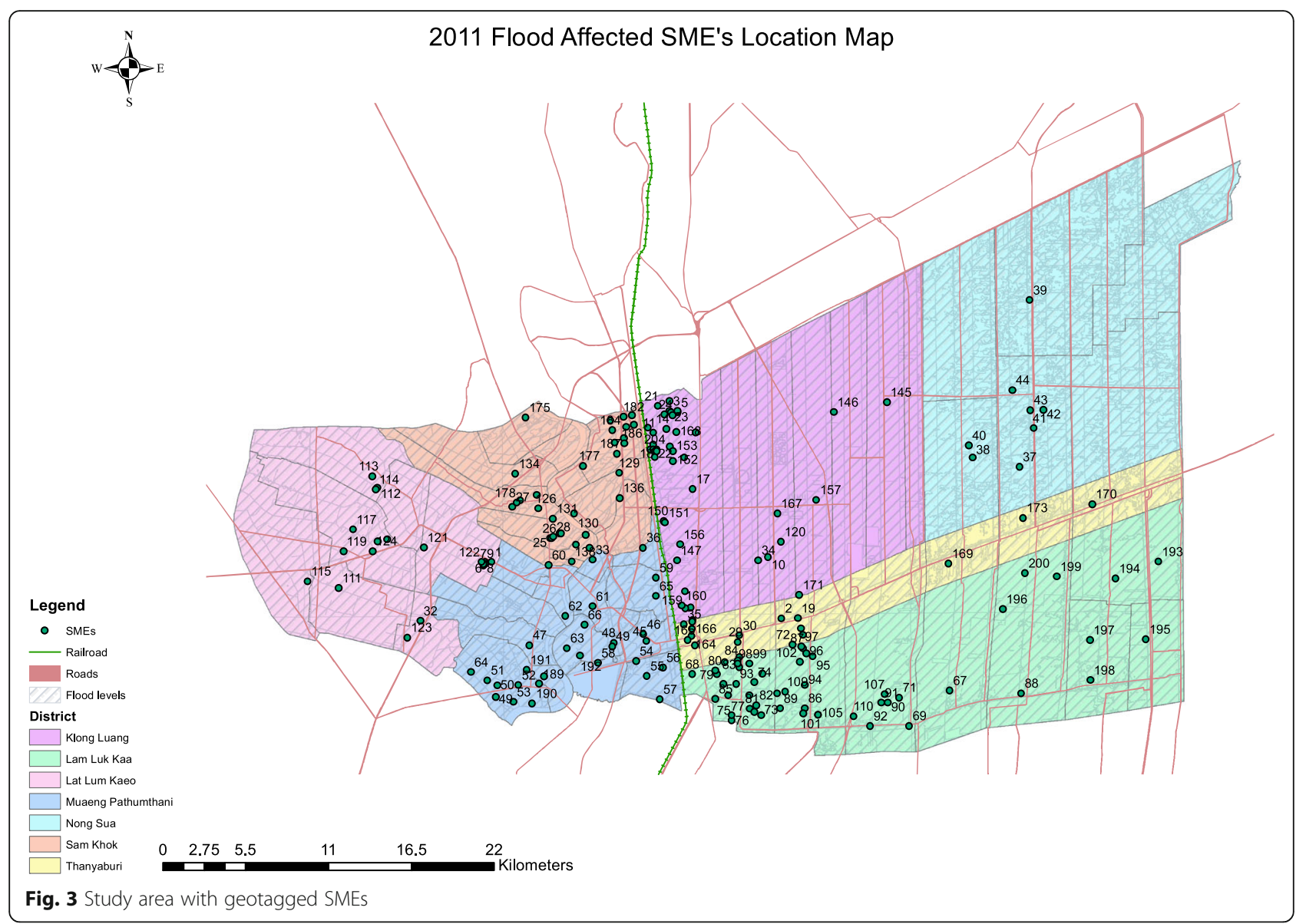

effective governance from ground level up to the national levels to provide for resilient and sustainable industrial sector in Thailand.

In order to understand the demographic and characteristics of the decision makers at the SME level, the endogenous factors such as gender, age and education were considered due to collective presence of these factors in the Thai SMEs as well in the previous literatures (Sydnor et al. 2017).

Quantitative and qualitative dataset which have been collected through survey questionnaire, key informant interviews and focus group discussion respectively. To collect the primary data, the population of affected SMEs were collected from the OSMEP department. There has been a total population of 400 SMEs in the study area (OSMEP 2014) and therefore, a sample of 177 SMEs were selected in this study (Yamane 1967) on the basis on their encounter and impacts from the 2011 floods. The sample SMEs were random purposively selected and survey questionnaires were extended to them which were returned with $100 \%$ accepted survey questionnaires. The secondary data collection involves various reports from the government organisation, international organisations and local level administrative bodies working for disaster management and recovery in the Thailand.

The collected data was analysed with $\mathrm{R}$ software and spatial analysis was done through geotagging the SMEs in ArcGIS software. However, in order to conceal the real identity and location of the SMEs, a unique id number was allotted to all the geotagged SMEs. The spatial econometrics approach has been adopted with multiple regression model.

\section{Results}

The results represent the spatially distributed locations of the DRTP variable, depicted the predominating high and low DRTP values (see, Fig. 3). It was further analyzed through these findings the functional relationship and spatial autocorrelation between DRTP vales at a specific location and at the neighbouring locations. In order to determine and ascertain geographical distribution and spatial global autocorrelation of DRTP the Moran's I test was performed. We used three different approaches of spatial interdependence (matrix $W$ ) as follows:

(1) inverse distance between neighboring observations; 
Table 1 Selected variables for analysing speed of disaster recovery

\begin{tabular}{lll}
\hline Variable & Description & Values \\
\hline $\begin{array}{l}\text { Dersonal variables } \\
\text { Gender }\end{array}$ & Disaster recovery time period (dependent variable) & In days \\
Age & Gender of the respondent (Management) & Male (1) or female (0) \\
Education & Age of the respondent (Management) & Years \\
Economic variables & Education of the respondent (Management) & Postgraduate (1) or not (0) \\
$\begin{array}{l}\text { Damage } \\
\text { Business }\end{array}$ & Economic damage amount & In thousands of dollars \\
Export & Type of the Business & Manufacturing (1) or Service (0) \\
Mitigation & Export by the SME & Yes (1) or No (0) \\
Insurance & Mitigation before 2011 floods & Yes (1) or No (0) \\
Loan & Insurance coverage before $2011 \mathrm{floods}$ & Yes (1) or No (0) \\
Risk & Pending bank loans due to $2011 \mathrm{floods}$ & Yes (1) or No (0) \\
Govt_res & Risk perception of floods before $2011 \mathrm{floods}$ & Yes (1) or No (0) \\
Govt_prep & Government response to $2011 \mathrm{floods}$ & Satisfactory (1) or not satisfactory (0) \\
BCP & Government preparation for $2011 \mathrm{floods}$ & Satisfactory (1) or not satisfactory (0) \\
\hline
\end{tabular}

Source: Field Survey conducted by Authors

(2) inverse distance-squared between neighboring observations;

(3) nearest neighbor with four different numbers of neighbors $(10,15,20$ and 25).

In all the above approaches, the $W$ matrix sum was standardized to unity (with row standardization) along with utilizing randomization method with 999 permutations.

The results (Table 2) depicted a positive significant spatial global autocorrelation across all spatial weights. It was found out that the DRTP in the Pathumthani nonrandomly distributed. This further depicted the spatial autocorrelation with higher DRTP being clustered together and vice versa for the lower DRTP. It was found that that W matrix and Moran's I test provided for high significant results. The results depicted that with highest $\mathrm{z}$-score the R-squared was highest and AIC was found to

Table 2 Spatial autocorrelation of DRTP, R-squared and AIC of SAR model for different conceptualizations of spatial relationships

\begin{tabular}{lllll}
\hline $\boldsymbol{W}$ & Moran's I & z-score & R-squared & AIC \\
\hline Inverse distance & 0.203 & $3.966(0.000)$ & 0.310 & 338.33 \\
Inverse distance squared & 0.083 & $1.621(0.053)$ & 0.286 & 344.2 \\
Nearest neighbor (10) & 0.115 & $3.959(0.000)$ & 0.297 & 341.44 \\
Nearest neighbor (15) & 0.081 & $3.571(0.000)$ & 0.295 & 341.91 \\
Nearest neighbor (20) & 0.069 & $3.667(0.000)$ & 0.290 & 343.07 \\
Nearest neighbor (25) & 0.053 & $3.287(0.001)$ & 0.294 & 342.24 \\
\hline
\end{tabular}

Note: $p$-values are given in brackets be relatively less (Refer Table 2)."Hence, we opted to use this W matrix to estimate spatial econometric model.

To consider the relation between DRTP and explanatory variables, we have specified a semi-logarithmic model where the dependent variable is the natural log of DRTP (lnDRTP). Table 3 shows the OLS-model where spatial interdependence among observations were not included for further consideration. Hence, the model overlooks of spatial autocorrelation and assumes the observations to be independent (Pace et al. 1998). The adopted model resulted in a value of 0.269 for the coefficient of the determinant (R-squared). In terms of the heterogeneity of the data, the value of 0.269 is acceptable as it not very high among the cross sectional data in research. The highest variance inflation factor (VIF) was found to be 1.662 which signifies that low multicollinearity in the data.

The Jarque-Bera test was conducted and it was found that normality of perturbations was also acceptable with statistical values being Chi-squared $=0.115, \mathrm{df}=2$ and $p$ value $=0.943$. Also, the homoscedasticity was acceptable, according to the Breusch-Pagan test with statistical values being $\mathrm{BP}=17.401, \mathrm{df}=13$ and $\mathrm{p}$-value $=0.181$. However, the perturbances are spatial autocorrelated, according to the Moran's I test of errors ( $I=0.164$, pvalue $=0.000)$. Therefore, we need to specify a spatial model which considers the relationship between InDRTP and locational factors, as SAR-model.

The R2-squared and AIC of SAR-model and SEMmodel are very similar. To conclude the best suited model amongst the SAR model or SEM, we conducted a 
Table 3 Models. Dependent variable: InDRTP

\begin{tabular}{|c|c|c|c|}
\hline & OLS-model & SAR-model & SEM-model \\
\hline const & $3.892(0.000)$ & $2.691(0.000)$ & $3.901(0.000)$ \\
\hline Damage & $0.0002(0.032)$ & $0.0002(0.010)$ & $0.0002(0.002)$ \\
\hline Gender & $0.031(0.767)$ & $0.030(0.759)$ & $0.026(0.784)$ \\
\hline Age & $0.006(0.139)$ & $0.007(0.0967)$ & $0.006(0.102)$ \\
\hline Education & $-0.270(0.085)$ & $-0.306(0.033)$ & $-0.262(0.061)$ \\
\hline Export & $0.119(0.315)$ & $0.093(0.394)$ & $0.076(0.471)$ \\
\hline Mitigation & $0.390(0.000)$ & $0.355(0.000)$ & $0.371(0.000)$ \\
\hline Insurance & $-0.294(0.022)$ & $-0.272(0.021)$ & $-0.306(0.010)$ \\
\hline Loan & $0.364(0.005)$ & $0.330(0.005)$ & $0.320(0.006)$ \\
\hline Risk & $-0.215(0.093)$ & $-0.216(0.066)$ & $-0.223(0.052)$ \\
\hline Govt_res & $0.551(0.001)$ & $0.520(0.001)$ & $0.516(0.001)$ \\
\hline Govt_prep & $-0.376(0.018)$ & $-0.322(0.027)$ & $-0.291(0.038)$ \\
\hline $\mathrm{BCP}$ & $-0.381(0.000)$ & $-0.321(0.000)$ & $-0.289(0.002)$ \\
\hline Business & $0.085(0.493)$ & $0.101(0.377)$ & $0.111(0.323)$ \\
\hline Wy & - & $0.275(0.001)$ & - \\
\hline Wu & - & - & $0.324(0.001)$ \\
\hline R-squared & 0.269 & 0.310 & 0.310 \\
\hline $\mathrm{AlC}$ & 346.39 & 338.33 & 338.17 \\
\hline Moran's I err & $0.164(0.001)$ & $0.010(0.922)$ & - \\
\hline LM-lag & $11.754(0.000)$ & - & - \\
\hline LM-err & $9.496(0.002)$ & - & - \\
\hline Robust LM-lag & $2.292(0.130)$ & - & - \\
\hline Robust LM-err & $0.036(0.847)$ & - & - \\
\hline$n$ & 177 & 177 & 177 \\
\hline
\end{tabular}

*: p-values are shown in brackets

classical analysis using LM statistics. In view of the LM and robust-LM statistics (Table 3), SAR was deemed more suitable than SEM.

In all the models, the explanatory variables: Damage, Mitigation, Insurance, Loan, Govt_res, Govt_prep and $\mathrm{BCP}$ were significant at least at $5 \%$, and their signs were as expected and Education is significant at $5 \%$ in the SAR-model and the sign was as expected. Semilogarithmic model allows investigating the relative impact of the explanatory variables. The coefficients $\beta_{k}$ of continuous variables were multiplied by 100 to interpret the percentage impact of this variable on DRTP. However, if explanatory variable is a dummy variable, the percentage effect on DRTP is equal to $100\left\{\exp \left(\beta_{k}\right)-1\right\}$ (Halvorsen and Palmquist 1980).

The explanatory variables are not depicted by the estimation of the coefficients in the SAR- model (Pace and LeSage 2009). Therefore, in order to accurately understand and analyze the coefficients of the SAR-model, calculations were performed for each of explanatory variable in terms of direct and indirect as well as total effect on the model as even marginal change would affect in each category of effects (Table 4). The explanatory variable in any neighbouring spatial location $j$ depicts effect of dependent variable (Debarsy et al. 2012). The spillover effect is also undertaken so that the logical consequences of the model are also included among the interpretations. This resulted in understanding and analyzing indirect effects through measuring spatial spillover effect among explanatory variable and dependent variable. Our model depicted around 35\% among direct effects.

In our SAR-model the direct, indirect and total impacts show the effect of explanatory variables on DRTP (see, Table 4). For instance, if the company $j$ dispose to continuity planning before 2011 floods $(\mathrm{BCP}=1)$ this reduces the disaster recovery time period in a $38.81 \%$ of company $j$. But if this decision is taken for neighbor companies, the disaster recovery time period, of this company $j$, is reduced in a $12.18 \%$ and the total reduction will be about $55.73 \%$. In our case, the indirect effect measures if the disaster recovery time period of one company depends on the decision of neighbors companies about continuity planning before the 2011 floods.

Therefore, spatial econometric modeling has been found to be more appropriate in comparison with the classical modeling. It shows that DRTP is spatially distributed according to certain configurations of agglomeration, signifying that companies from neighboring locations were found to having similar actions and performance.

The spatial interdependence among the flood vulnerable SMEs provides for robust foundations towards framing the resilient government policies to reduce the DRTP in future. It is essential for the policy makers to incorporate such findings for designing and implementing the actual needs and requirement of the SMEs for

Table 4 Direct, indirect and total effects

\begin{tabular}{llll}
\hline & Direct & Indirect & Total \\
\hline Damage & $0.00017(0.007)$ & $0.00006(0.079)$ & $0.00023(0.011)$ \\
Gender & $0.030(0.830)$ & $0.011(0.815)$ & $0.041(0.823)$ \\
Age & $0.007(0.058)$ & $0.002(0.125)$ & $0.009(0.059)$ \\
Education & $-0.313(0.026)$ & $-0.110(0.098)$ & $-0.422(0.030)$ \\
Export & $0.095(0.421)$ & $0.033(0.489)$ & $0.128(0.431)$ \\
Mitigation & $0.362(0.000)$ & $0.127(0.059)$ & $0.489(0.001)$ \\
Insurance & $-0.278(0.030)$ & $-0.097(0.113)$ & $-0.375(0.038)$ \\
Loan & $0.337(0.006)$ & $0.118(0.097)$ & $0.455(0.010)$ \\
Risk & $-0.221(0.084)$ & $-0.077(0.186)$ & $-0.298(0.096)$ \\
Govt_res & $0.531(0.000)$ & $0.187(0.053)$ & $0.718(0.001)$ \\
Govt_prep & $-0.329(0.021)$ & $-0.116(0.129)$ & $-0.445(0.030)$ \\
BCP & $-0.328(0.000)$ & $-0.115(0.041)$ & $-0.443(0.000)$ \\
Business & $0.103(0.376)$ & $0.036(0.436)$ & $0.139(0.385)$ \\
\hline
\end{tabular}

Note: $p$-values in brackets 
disaster risk reduction. The SMEs tend to perceive the flood or disaster risk as unforeseen and uncertain events. Therefore, the urgency towards investing in the disaster mitigation process becomes secondary expenses for the enterprises.

\section{Discussions}

The personal variables inclusive of Gender, Age and Education level of the SME's decision making tend to provide for innovative analysis of the flood scenario. Gender of decision makers has been considered to be important factor as female and minority decision makers tend to end up with longer DRTP (Marshall et al. 2015). Although Gender variable in our models is not significant, it signifies that there is not significant difference between management men and women of SME. This was found to be similar among the respondents during the Key informant interviews. One of the respondents from the provincial government disaster mitigation office adds,

"Women in Thailand are very hardworking and responsible. This tends to increase their trauma and responsibilities towards the SME, their household as well their employee's wellbeing. It is unfortunate that even after giving their best, the women find it difficult to reduce the flood recovery time period within their SME as well as among neighbouring SMEs."

Similarly, the variable age plays a vital role in coping up with a disaster event and tend to reflect upon the DRTP of SMEs among Thailand. Previous researchers presented age as an important factor determining the duration of DRTP after the devastating floods in 2010 in Pakistan (Asgary et al. 2012; Asgary et al. 2020). The respondent from the Ministry of Social Development emphasized that age contributes to the risk perception and acceptability to invest in disaster mitigation measures to reduce flood risks. He adds,

"I must make a statement that in Thailand the veterans are reluctant to invest in flood mitigation initiatives. They have seen floods before but they do not analyse or understand the magnitude of the floods and its long term implications. The younger generations tend to learn from neighbouring SMEs and try to adopt the mitigation during and after floods."

Among the significant personal variables, education tends to be crucial for reducing the DRTP and coping up with the disaster events. Previous studies also emphasized upon positive relation between education level and
DRTP (Sydnor et al. 2017), however, our findings confirm the significance of education among neighbouring SMEs as well. A respondent from the district level commerce department adds,

"The SME managers/owners are very receptive towards the flood mitigation measures. They understand the implications of facing the floods in all socio-economic terms. The college degree really enables them to understand, analyse and reduce the disaster impact time period (DRTP)."

The Thai SMEs are a crucial part of various local, national and international supply chain management systems. 2011 floods impacts were felt all through these supply chains and repercussions were felt in almost every part of the world. For instance, the hike in the prices of hard disks in USA due to the impacts upon the SMEs contributing towards the Western Digital company to manufacture hard disk (Sheffi 2020). One of the respondents among the leading instant noodle company in Thailand who suffered losses in his supply chain both in terms of the raw materials supply and end product delivery to various parts of the word. He adds

"Our supply chain management completed failed without the disaster recovery planning. Our suppliers did not deliver raw materials in time and we were inundated for a period of 55 days so could not deliver our noodle orders. Now we think of investing in flood preparedness or prevention measures for future."

The explanatory variables were also found to be significant, this is in line with several previous literatures (Asgary et al. 2012; Li and Hong 2019). Among them, Mitigation from floods have been one of the most crucial in terms of ascertaining the speed or duration of the DRTP among vulnerable SMEs. Among the respondents, there was a lack of adopted mitigation measures before the 2011 floods. This was found to be in line with the various other factors involving the perceived risk and decisions taken by the neighbouring SMEs (Shafi et al. 2020). It was found that flood mitigation measures (structural) were in line with the neighbouring SMEs. Therefore, it resulted in accumulation of several dykes leading to increased flood water levels in the already flood inundated areas. The decisions made by neighbouring SMEs resulted in higher impacts on them as a whole. One of the SME owner adds,

"Everyone was protected themselves with dykes, SME with sand bags and Large enterprises with concrete dykes but lack of cooperation among us 
led to several dykes wasted, rather increased the flood (Levels)."

Such mitigation measures were observed throughout the central region of Thailand making it difficult for the flood mitigation at the government level. It led to unprepared SMEs being exposed to higher flood water levels and magnitude of the floods. Despite the individual efforts the flood mitigation resulted in a failure without adequate cooperation between the SMEs and the government channels.

On the other hand, non-structural measures were scarcely adopted among the flood vulnerable SMEs in the study area. It was observed that non-structural measures such as insurance, which would have reduced the monetary losses and mental trauma and stress among the flood affected SMEs, was not adopted by most of the vulnerable SMEs. The reason for lack of adoption of Insurance as a flood mitigation measure and reducing the DRTP among the vulnerable SMEs was higher rate of insurance premium and lack of trust and collaboration between the SMEs and the insurance companies (Verbano and Venturini 2013). One of the respondents from flood affected SME added,

"Insurance prior to 2011 floods was an expensive and not so urgent expense for all of us. The premium is high; we have no clue if we would be flooded or be paid in time after the floods. Insurance companies never come to us for flood insurance they only come life insurance in terms of individuals or group employees."

Similarly, the leading insurance company in the study area was not surprised on non-adoption of Insurance as a mitigation strategy from flood events. She added,

"We never discussed flood insurance whenever we reach out to them for insurance. They are never interested as we understand the high premium rates and no compulsion from government organizations."

Therefore, Insurance need to be a compulsory measure to be implemented through government channels towards the vulnerable SMEs. However, to be supportive towards those SMEs, the government may provide subsidies in order to generate a motivation towards adopting insurance as a non- structural flood mitigation measure.

The financial security brings about the less stressful situation for the flood affected SMEs, which in turn enhances their capacities to recover faster from disaster scenarios. 2011 floods were an eye-opener for the SMEs as they understood the requirement of the fast and adequate financial assistance both from government as well as the financial institutions. However, it was found to be really difficult for around $85 \%$ of the companies to procure adequate finances for faster recovery from the 2011 floods. This was in line with the findings from various SME promotion and financial institution's schemes to assist the SMEs (OSMEP 2014), as lack of awareness deprived the affected SMEs from availing the loans or financial assistance. One of the banking official adds,

"No matter how many schemes we have to assist the flood affected SMEs, without adequate communication and sharing the information they will not be able to fully utilize the financial loan and security features including the promotion of entrepreneur."

Another major factor was the misperception of risk among the vulnerable SMEs. It was almost negligible possibility for the SMEs to be flooded at such high magnitude. Therefore, this unawareness and misinterpretation of risk perception led to escalating losses and damages during the post 2011 floods. With absence of awareness of actual risk, the perceived risk was lower and resulted in long term impacts of 2011 floods. One of the respondents from the SME residing among vulnerable areas adds,

"It is essential to know that we are at risk. Why else will we be spending on disaster preparedness, mitigation or recovery? It takes ample resources including physical and financial to be dealt with these uncertain events. However, our perception has changed after the experience of 2011 floods. It is easier to be prepared than managing post flood losses."

The findings shed light upon the mismanagement of the 2011 floods by the then government and several previous research provide the evidence of the same. The response from the government towards the unforeseen factors contributing towards the magnitude of the 2011 floods let to the escalating losses. It was found that neighbouring SMEs found it problematic to collaborate with the local level government's relief and recovery departments due to their strict top to bottom approach of governance in Thailand. There were sufficient resources with several departments at the local level, however without the prior approval from higher authorities the local department were not able to provide immediate and prompt relief and recovery assistance towards the SMEs. The major impact was borne by the SMEs in the remote location of the Pathumthani province which are scattered throughout the province. In words of respondent from the local fire station service, he adds, 
"There were motorised boats and big fire trucks available with us and we received the call for assistance from the nearby SMEs. However, we are not allowed to assist without the prior approval from the provincial authorities as we were kept on standby for more than 3 days."

Such attitudes led to unused resources at the local level. Similarly, the officer from the postal department in the rural area, she said,

"I was shocked at the amount of trucks and minivans which we kept in our facilities. With proper planning we could assisted the companies in the nearby areas. At least to move their movable assets to higher grounds or at least to the less flood (vulnerable) locations."

Likewise, in the terms of the preparedness of the government, the government was not prepared for the floods, in terms of managing flood waters, utilizing resources, coordinating among various government bodies and departments, providing timely early flood warning to the local SMEs, deal with the uneven flood water heights, security issues including inundation of crocodile farm and most of crocodiles being freed into the flood waters, danger from the contaminated flood water borne diseases and animal attacks also including monitor lizards, dogs, snakes as well.

One the provincial disaster management official admits that there was mismanagement at the part of the government at the national level. As the disaster management framework is already existing in Thailand before 2011 floods the overall impacts could be reduced with adequate preparedness at the government level. He adds,

"I, Myself being at the decision making position, was unable to deliver the best of my ability. There was tremendous pressure from high authorities and ample demands from the subordinate levels. I wanted and could have assisted better if at least the provincial level had some urgent decision making authority. All this increase the stress and reduces are capacities and capabilities to deal with the floods."

The neighbouring SMEs were abandoned and left to manage their enterprises on their own. However, this changed with government relief measures after the floods with assisting in terms of financial aid provided by the government agencies. This included coverage of $75 \%$ of the wages to be paid to the registered employees of the SMEs. Though the non-registered employees struggled to survive the aftermath of 2011 floods.
One of the major planning component, Business continuity Planning (BCP) was found to be missing with most of these 2011 flood affected SMEs. This is in line with the fact that as one SME adopts the BCP and the neighbouring SMEs themselves try to collaborate and plan the similar BCPs for their enterprises. This found in one the flood vulnerable region in the study area. However, such planning should be mandatory with the compulsion of the government as well among the SMEs.

In order to adopt the structural, non-structural flood mitigation measures and planning for business continuity during and after disasters, should be a responsibility of the SMEs. Although, the external aid and assistance was found to be negligible during the 2011 floods, SMEs could have adopted some of the measures before the 2011 floods to be prepared for the floods. This is in line with the fact that unawareness and inaccurate risk perception among the vulnerable SMEs led to enormous losses. Similarly, the government schemes such as providing benefits and assistance to provide for staff's $75 \%$ of salary was not provided to all SMEs. One of the respondent from SME added, respondent added,

"I am over fifty years of age, it was already too tiresome for me to manage personal and SME. The government did not help in any way. The schemes they had were not informed to me. I came to know through my neighbouring companies. They can at least share to each group of SMEs (Cluster). It was very hard for me continue after 2011 floods."

These findings and observation provide for the importance of utilizing the spatially interdependent SMEs in the flood affected region. Through the adequate disaster recovery planning and improving the governance mechanisms to provide the relief and recovery assistance towards the vulnerable SMEs, the DRTP could be reduced for the future events.

\section{Conclusions}

The disaster recovery period is crucial for determining the future growth and development of an SME after being encountered by a natural disaster. The major factors that determine and reduce DRTP includes the impact of disaster resilience, mitigation and planning at the SME level as well as the government level. The absence of accurate perception of actual risk, flood insurance and disaster management planning prior to the 2011 floods had contributed to the severity of the impacts during the 2011 floods.

. In this paper we have found that due to the disaster recovery time period (DRTP) of a SME depends on their neighbors, to improve the DRTP of a SME will improve the DRTP of neighbors. Based on our findings, the 
following are the primary stakeholders' recommendations in ascertaining and minimizing the Disaster Recovery Period. On the other hand, improving some factors (Education, Mitigation, Government response and Business continuity planning) of my neighbors also improving my DRTP.

Based on our findings, the following are the primary stakeholders' recommendations in ascertaining and minimizing the Disaster recovery Period:

Recommendations towards the SMEs:

- The neighboring SMEs must collaborate to adopt flood preparedness and mitigation planning to adapt to the flood vulnerability.

- On their level, SMEs must include structural, nonstructural measures to curtail the damages from the flood.

SMEs need to design and implement Business Continuity Planning, Disaster Recovery Planning to reduce the Disaster Recovery Period adequately.

Recommendations towards the Government

The government must cater to the actual needs of the SMEs in various levels of their vulnerabilities. They can achieve the targeted goals through useful vulnerability mapping and zonation for flood vulnerable areas.

- There must be an adequate Top to Bottom (existing) and Bottom to Top (to be implemented) approach towards disaster management through the already existing disaster management framework in Thailand.

- The government must impose mandatory flood insurance, especially in flood vulnerable areas, Disaster Recovery, and Business Continuity planning, and the local government levels to reduce the Disaster Recovery Period.

- The government must provide awareness, timely early flood warnings, and mock drills to enable the disaster management framework's actual implementation and reduce vulnerabilities from future flood events.

Recommendations towards the Non-Government Organizations and Financial Institutions

- The Non- Government Organizations (NGOs) and the financial institutions must collaborate with the local level neighboring SMEs to pool all the resources during their relief, rehabilitation, and recovery operations during and after flood events.

- The financial institutions must ensure proper and adequate reach of their SMEs' policies to assist and reduce the Disaster Recovery Period accurately.
The paper is useful and paves the way for future research to be carried out for middle-income developing countries that encounter natural disasters and their adverse impacts on business enterprises, mainly neighboring and spatially interdependent SMEs. The strategic model could be adopted and applied for other natural disasters, including earthquakes, Tsunami, landslides, avalanches, and forest fires. To achieve resilience and sustainability among the vulnerable SMEs, both the neighboring SMEs and the government channels need to collaborate and provide sustainable growth and development of the economy as a whole.

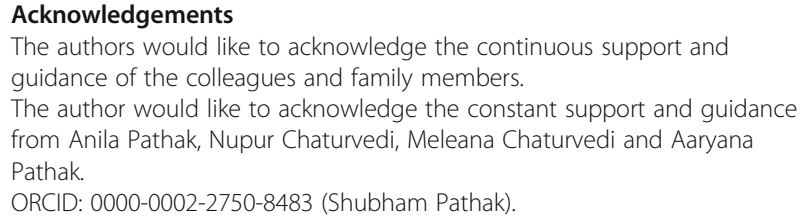

\section{Authors' contributions}

The authors contributed collectively towards the manuscript. However, the detailed contributions among various research inputs included:

Conceptualization- 1st Author; Methodology-1st and 2nd author; Data collection; 1st author; Data analysis: 1st and 2nd author; Write up- 1st author; supervision and revisions 1 st and 2 nd author. The author(s) read and approved the final manuscript.

\section{Authors' information}

1. Dr. Shubham Pathak is a Lecturer and Program Coordinator for Logistics analytics and supply chain management program at Walailak University International College, Thailand. He received his PhD. from Disaster Preparedness, Mitigation and Management (DPMM) at Asian Institute of Technology (AIT), Thailand. He worked on several projects for Disaster Risk Reduction Program of UNDP under Govt. of Uttarakhand, Dehradun, India. He has experience among GIS and remote sensing technologies, agriculture, business, gender projects. He has academic publications in journals including International Journal of Disaster Risk Reduction, Development in Practice and Natural Hazards researching in India, Thailand, Timor Leste, Spain and Nigeria.

2. Jorge Chica Olmo is a university Professor at Departamento de Métodos Cuantitativos para la Economía y la Empresa (Department of Quantitative Methods for Economics and Managment), Facultad de Ciencias Económicas y Empresariales (Faculty of Economics and Business), Universidad de Granada (University of Granada), Spain. He specialises in Spatial Econometrics, GIS, Economics, Geography, Rural Development and Business studies. He has several lading publications and is keen on research oriented approach of university teaching.

Funding

This research was conducted and funded by the authors.

Availability of data and materials

The datasets generated and/or analysed during the current study are available from the corresponding author on reasonable request.

\section{Declarations}

Competing interests

The authors declare that they have no competing interests.

\section{Author details}

'Logistics Analytics and Supply Chain Management, Walailak University International College, Walailak University, Tha Sala, Nakhon Si Thammarat, Thailand. ${ }^{2}$ Departamento de Métodos Cuantitativos para la Economía y la Empresa, Universidad de Granada, Granada, Spain. 
Received: 9 August 2020 Accepted: 24 March 2021 Published online: 06 April 2021

\section{References}

(OSMEP), Office of SMEs Promotion. 2011. The 2nd SMEs Promotion Plan (20072011)

Anselin L (1988) Spatial econometrics: methods and models. Kluwer Academic Publishers, Dordrecht. https://doi.org/10.1007/978-94-015-7799-1

Anselin, Luc, Anil K. Bera, Raymond Florax, and Mann J. Yoon. 1996. "Simple diagnostic tests for spatial dependence". Reg Sci Urban Econ 26 (1): 77-104. https://doi.org/https://doi.org/10.1016/0166-0462(95)02111-6

Asgary, Ali, Muhammad Imtiaz Anjum, and Nooreddin Azimi. 2012. "Disaster recovery and business continuity after the 2010 flood in Pakistan: case of small businesses". Int J Disaster Risk Reduct 2: 46-56. https://doi.org/https:// doi.org/10.1016/j.jjdrr.2012.08.001

Asgary, Ali, Ali Ihsan Ozdemir, and Hale Özyürek. 2020. "Small and medium enterprises and global risks: evidence from manufacturing SMEs in Turkey". Int J Disaster Risk Sci 11 (1): 59-73. https://doi.org/https://doi.org/10.1007/s13 753-020-00247-0

Coquet, Marie, Denis Mercier, and Ghozlane Fleury-Bahi. 2018. "Individuals' perceptions of areas exposed to coastal flooding in four French coastal municipalities: the contribution of sketch mapping". Geoenviron Disasters 5 (1). https://doi.org/https://doi.org/10.1186/s40677-018-0107-3

Cravo, Tulio A., Bettina Becker, and Adrian Gourlay. 2015. "Regional growth and SMEs in Brazil: a spatial panel approach". Reg Stud 49 (12): 1995-2016. https://doi.org/https://doi.org/10.1080/00343404.2014.890704

Debarsy N, Ertur C, Lesage JP, Debarsy N, Ertur C, Lesage JP, Interpreting Dynamic, Space-time Panel Data, Lesage JP (2012) Interpreting Dynamic Space-Time Panel Data Models To Cite This Version : HAL Id : Hal-00525740 Document de Recherche $n^{\circ}$ 2010-22 « Interpreting Dynamic Space-Time Panel Data Models » Cem ERTUR Nicolas DEBARSY, pp 158-171

Elhorst JP, Gross M, Tereanu E (2018) Spillovers in Space and Time: Where Spatial Econometrics and Global VAR Models Meet. ECB Working Paper. Vol. 0

Elhorst J.P. 2014. "Linear Spatial Dependence Models for Cross-Section Data." In Spatial Econometrics. SpringerBriefs in Regional Science. Berlin, Heidelberg: Springer Berlin Heidelberg. https://doi.org/https://doi.org/10.1007/978-3642-40340-8_2

Ezgi O (2016) Lessons learned from businesses to ensure community level recovery in a Postdisaster period: case from Adapazari, Turkey. Natural Hazards Rev 17(1):1-12.

Government, Royal Thai, and The World Bank. 2012. "Thailand Flooding 2554 Rapid Assessment for Resilient Recovery and Reconstruction Planning"

Halvorsen, Palmquist R (1980) The interpretation of dummy variables in Semilogarithmic equations. Am Econ Rev 70(3):474-475

Haraguchi, Masahiko, and Upmanu Lall. 2015. "Flood risks and impacts: a case study of Thailand's floods in 2011 and research questions for supply chain decision making." Int J Disaster Risk Reduct 14 (January 2012): 256-272. https://doi.org/https://doi.org/10.1016/j.ijdrr.2014.09.005

Horney, Jennifer, Matt C Simon, and Kristen Ricchetti-masterson Philip. 2016. "Resident perception of disaster recovery planning priorities." Int J Disaster Resilience Built Environ 7 (4). https://doi.org/https://doi.org/10.1108/JJDRBE09-2014-0068, 330, 343

Khaikhan, Ladawan, and Helen James. 2019. "Population, Development, and the Environment: Challenges to Achieving the Sustainable Development Goals in the Asia Pacific." Population, Development, and the Environment: Challenges to Achieving the Sustainable Development Goals in the Asia Pacific, no. February: 1-440. https://doi.org/https://doi.org/10.1007/978-981-13-2101-6

Khan, Mohammad Aftab Uddin, and Mohammad Amir Sayem. 2013. "Understanding recovery of small enterprises from natural disaster". Environ Hazards 12 (3-4): 218-239. https://doi.org/https://doi.org/10.1080/17477891.2 012.761593

LeSage JP (2008) An introduction to spatial econometrics. Rev Econ Ind 123:1944

Lesage JP (1999) Applied Econometrics Using MATLAB

Li, Fan, and Jingke Hong. 2019. "A spatial correlation analysis of business operating status after an earthquake: a case study from Lushan, China." Int J Disaster Risk Reduct 36 (March): 101108. https://doi.org/https://doi.org/10.101 6/j.j.jdr.2019.101108

Marshall, Maria L, Linda S. Niehm, Sandra B. Sydnor, and Holly L Schrank. 2015. "Predicting small business demise after a natural disaster: an analysis of pre- existing conditions." Nat Hazards 79 (1): 331-354. https://doi.org/https://doi. org/10.1007/s11069-015-1845-0

Moran, P. A. P. 1950. "Notes on continuous stochastic phenomena." Biometrika 37 (1/2): 17. https://doi.org/https://doi.org/10.2307/2332142

OSMEP. 2014. "Direction and measures of SMEs promotion"

OSMEP (2018) Gross Domestic Product of Thai SMEs, pp 1-8 http://www.sme.go. th/upload/mod_download/download-20180912110443.pdf

Pace R, LeSage J (2009) Introduction to spatial econometrics. Taylor \& Francis Group

Pace RK, Barry R, Sirmans CF (1998) Spatial statistics and real estate. J Real Estate Financ Econ 17(1):5-13. https://doi.org/10.1023/A:1007783811760

Pathak, Shubham, and Mokbul Morshed Ahmad. 2016. "Flood recovery capacities of the manufacturing SMEs from floods: a case study in Pathumthani Province, Thailand." Int J Disaster Risk Reduct 18: 197-205. https://doi.org/ https://doi.org/10.1016/j.ijdrr.2016.07.001

Rikimaru, Yuuki, and Ritei Shibata. 2017. "Non-Identifiability of simultaneous spatial autoregressive model and singularity of fisher information matrix." Int J Stat Probability 5 (4): 31. https://doi.org/https://doi.org/10.5539/ijsp.v6n4p31

Shafi, Mohsin, Junrong Liu, and Wenju Ren. 2020. "Impact of COVID-19 pandemic on micro, small, and medium-sized enterprises operating in Pakistan." Res Global 2: 100018. https://doi.org/https://doi.org/10.1016/j.resglo.2020.100018

Sheffi Y (2020) Who gets what when supply chains are disrupted? MIT Sloan Manag Rev:1-10 https://sloanreview.mit.edu/article/who-gets-what-whensupply-chains-are-disrupted/

Suvittawat A (2019) Entrepreneurial passion domains of small and medium enterprises (SMES): a case study of lower northeastern, Thailand. Int J Entrep 23(1):4675

Sydnor, Sandra, Linda Niehm, Yoon Lee, Maria Marshall, and Holly Schrank. 2017. "Analysis of post-disaster damage and disruptive impacts on the operating status of small businesses after hurricane Katrina." Nat Hazards 85 (3): 16371663. https://doi.org/https://doi.org/10.1007/s11069-016-2652-y

Verbano C, Venturini K (2013) Managing risks in SMEs: a literature review and research agenda. J Technol Manag Innov 8(3):186-197

Wall, Melanie M. 2004. "A close look at the spatial structure implied by the CAR and SAR models." J Stat Plan Inference 121 (2): 311-324. https://doi.org/ https://doi.org/10.1016/S0378-3758(03)00111-3

Xiao, Yu, and Walter Gillis Peacock. 2014. "Do Hazard mitigation and preparedness reduce physical damage to businesses in disasters? Critical role of business disaster planning." Nat Hazards Rev 15 (3): 04014007 . https://doi. org/https://doi.org/10.1061/(asce)nh.1527-6996.0000137

Yamane T (1967) Statistics, an introductory analysis, 2nd edn. Harper and Row, New York

Yu, Xi, and Yandong Tang. 2017. "A critical review on the economics of disasters." J Risk Anal Crisis Response 7 (1): 27. https://doi.org/https://doi.org/10.2991/jra rc.2017.7.1.4

Zhang, Lei. 2016. "Flood hazards impact on neighborhood house prices: a spatial Quantile regression analysis." Reg Sci Urban Econ 60: 12-19. https://doi.org/ https://doi.org/10.1016/j.regsciurbeco.2016.06.005

Zhang, Yang, Michael K Lindell, and Carla S Prater. 2009. "Vulnerability of community businesses to environmental disasters." Disasters 33 (1): 38-57. https://doi.org/https://doi.org/10.1111/j.1467-7717.2008.01061.x

\section{Publisher's Note}

Springer Nature remains neutral with regard to jurisdictional claims in published maps and institutional affiliations. 